\title{
65. Marisome from Glycine and Acidic, Basic, and Aromatic Amino Acids in a Modified Sea Medium
}

\author{
By Hiroshi Yanagawa and Fujio Egami \\ Mitsubishi-Kasei Institute of Life Sciences, \\ 11 Minamiooya, Machida-shi, Tokyo 194
}

(Communicated by Shiro AKaborI, M. J. A., Sept. 12, 1978)

It is biochemically and paleontologically accepted that the origin and early evolution of life on the Earth took place in the primeval sea about $3 \times 10^{9}$ years ago. The essential components of a living organism must have been already formed abiogenetically and accumulated in the sea by that time and fundamental characters of organisms have been acquired under the primeval sea condition. However, how did the first protocell appear in the primeval sea? The appearance of complex phase-separated systems in aqueous solution of high molecular weight-compounds is certainly required for development of protocells. The best known examples of such phase-separated multi-molecular systems are coacervate droplets $^{1)}$ and proteinoid microspheres. ${ }^{2}$

In the course of research of chemical evolution in modified sea medium, we have recently found that protocell-like structures, marigranules ${ }^{3), 4)}$ are produced from glycine and acidic, basic, and aromatic amino acids and separated out after long incubation. The marigranules consisted of elastin-like polymers with molecular weights of 1,800 , $6,900,10,000$, and 82,000 , which had one peptide bond per three amino acid residues. In addition, we have recently observed that highly organized particles are phase-separated from concentrated aqueous solution of freeze-dried powder prepared from the reaction mixture containing glycine and acidic, basic, and aromatic amino acids in modified sea medium and we have termed the particle "marisome". Some properties of marisome are distinct from those of marigranules and marisome appears to be a precursor of marigranules. In the present paper, we report the structures and some properties of the marisome.

Modified sea medium ( $\mathrm{pH} 5.2$ ) contained $0.01 \mathrm{M}$ magnesium sulfate, $0.01 \mathrm{M}$ calcium chloride, $0.01 \mathrm{M}$ dibasic potassium phosphate and $0.1 \mathrm{mM}$ each of six transition metal ions $\left(\mathrm{Fe}\left(\mathrm{NO}_{3}\right)_{3}, \mathrm{Na}_{2} \mathrm{MoO}_{4}\right.$, $\mathrm{ZnCl}_{2}, \mathrm{Cu}\left(\mathrm{NO}_{3}\right)_{2}, \mathrm{CoCl}_{2}$, and $\mathrm{MnCl}_{2}$ ). Glycine (final concentration $0.05 \mathrm{M})$ and L-glutamic acid, L-aspartic acid, L-lysine, L-arginine, 
L-histidine, L-phenylalanine, L-tryptophan and L-tyrosine (each $5 \mathrm{mM}$ ) were added to the medium. The mixture (1.6 l) was kept at $105^{\circ} \mathrm{C}$ under nitrogen atmosphere. After 8 weeks the reaction mixture was filtered on $0.2 \mu \mathrm{m}$ pore size of Nuclepore membrane. The precipitate contained marigranules. On the other hand, the filtrate was freeze-dried. The yellowish brown freeze-dried powder was dissolved with distilled water and allowed to stand in boiling water for $10 \mathrm{~min}$. Then, the solution was filtered on $0.03 \mu \mathrm{m}$ pore size of Nuclepore membrane. When the reddish brown filtrate was allowed to stand at room temperature, a number of marisomes were phaseseparated. For scanning electron microscopy, the suspension of the marisome was allowed to settle on clean glass coverslips, which were gently dried at room temperature. Dried specimens were coated with platinum: palladium $(80: 20)$ in an Eiko IB-3 ion coater. Specimens were examined in a JEM 100C-ASID.

Fig. 1 shows ubiquitous marisome observed with scanning electron microscopy. The marisome had spherical structure of $2-4 \mu \mathrm{m}$ in diameter. When marisome was transferred to distilled water, it burst up immediately. Consequently, marisome was found to be an empty particle with soft envelope by scanning electron microscopic observation (Fig. 2). Marisome was completely solubilized with ethanol, 1\% sodium dodecylsulfate, and 1\% Triton X-100. Fig. 3

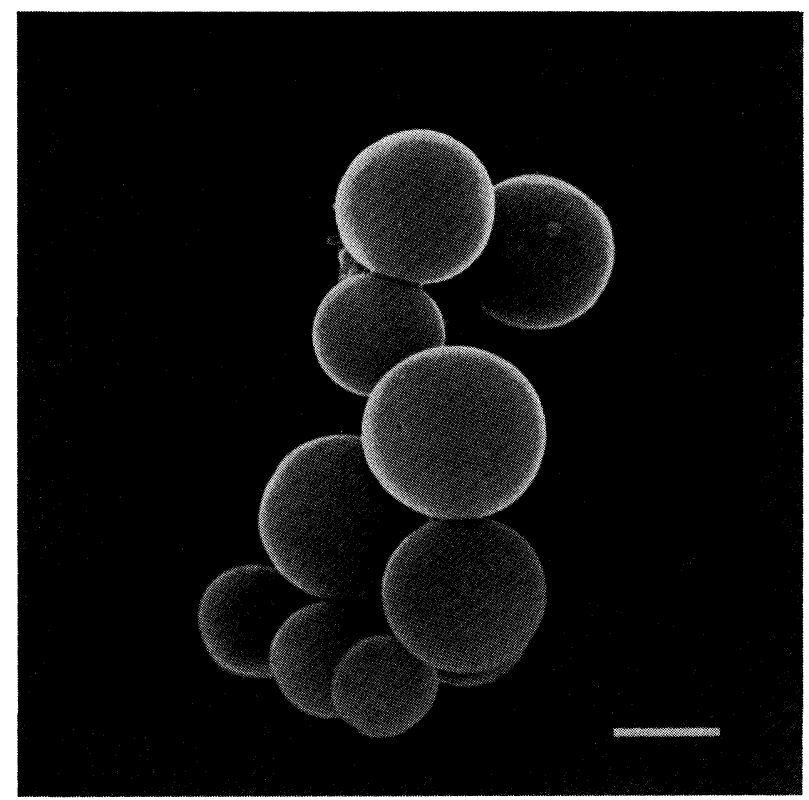

Fig. 1. Scanning electron micrograph of marisome (scale bar, $2 \mu \mathrm{m})$. 


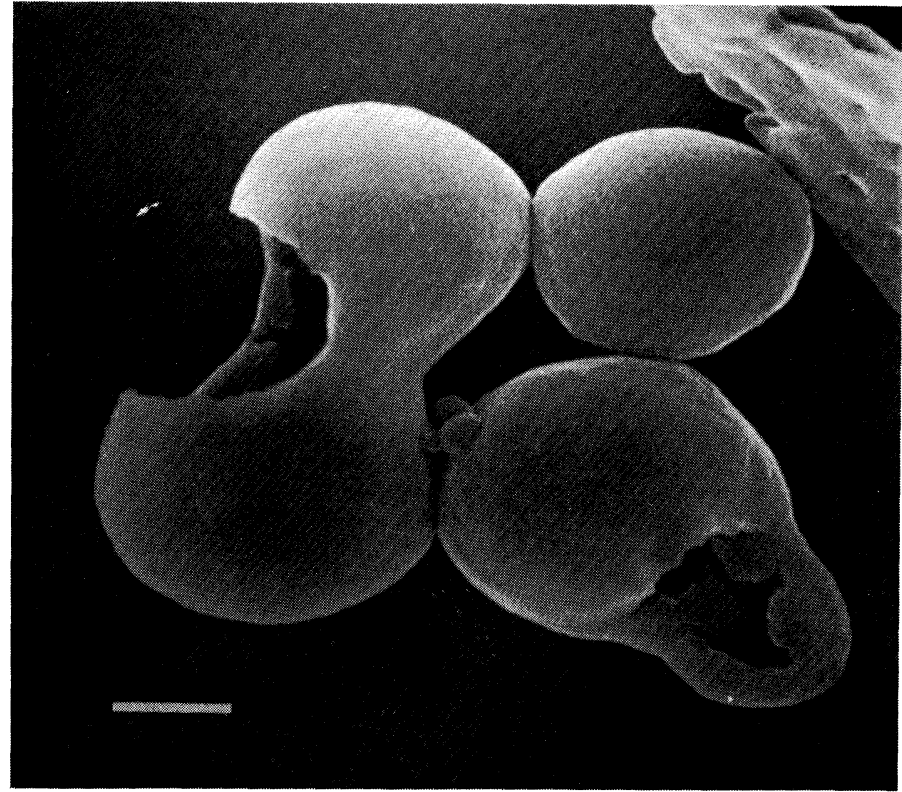

Fig. 2. Scanning electron micrograph of burst marisome with distilled water (scale bar, $1 \mu \mathrm{m}$ ).

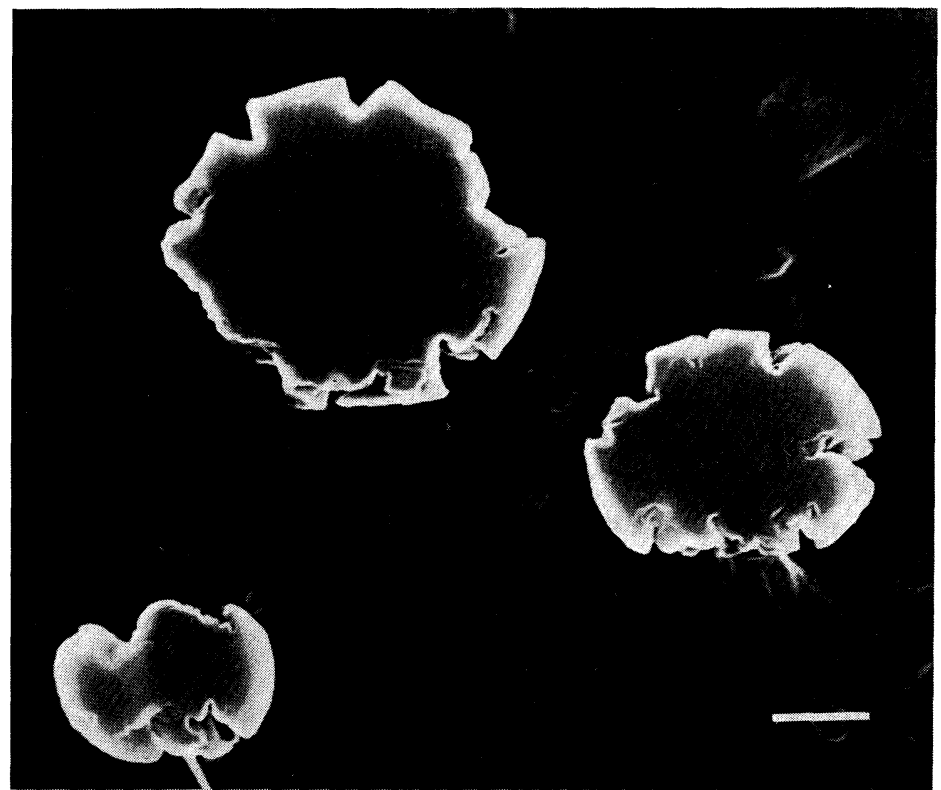

Fig. 3. Scanning electron micrograph of marisome treated with $1 \%$ Triton X-100 (scale bar, $2 \mu \mathrm{m}$ ). 


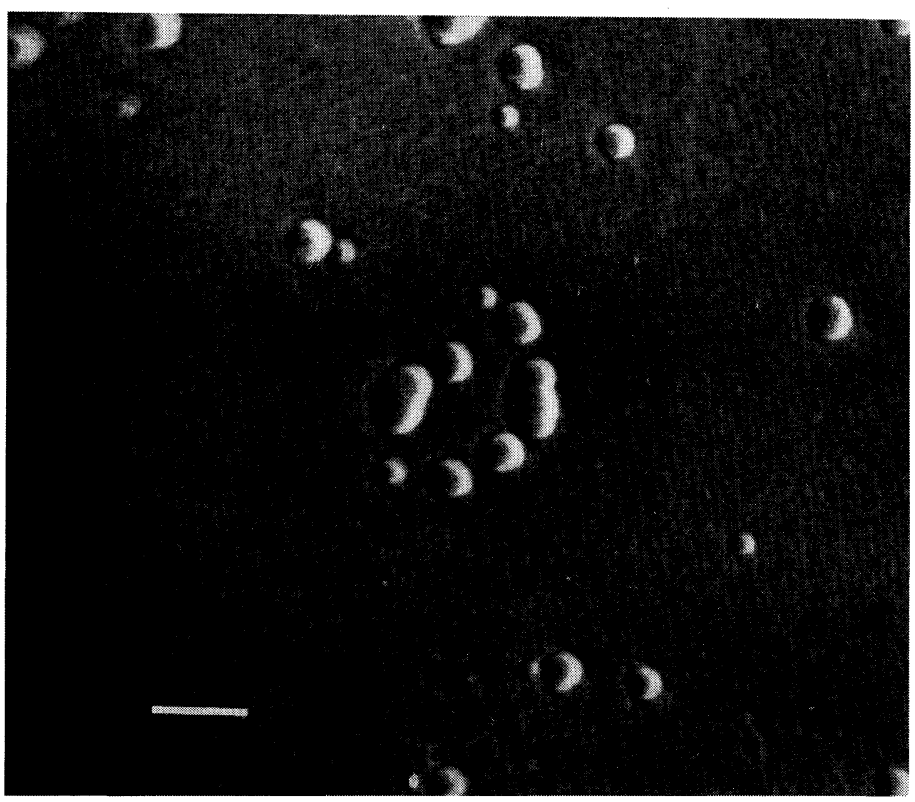

Fig. 4. Scanning electron micrograph of phase-separated marisome by dialysis against distilled water of ethanol solubilized marisome (scale bar, $0.2 \mu \mathrm{m}$ ).

shows marisome treated with $1 \%$ Triton $\mathrm{X}-100$. This photograph also shows that the interior of marisome is empty. However, structure of marisome was not changed by treatment with $1 \mathrm{~N} \mathrm{KOH}$ and $1 \mathrm{~N} \mathrm{HCl}$ solutions. The solubilized marisomes with ethanol, sodium dodecylsulfate, and Triton X-100 were phase-separated again by dialysis against water (Fig. 4). It was proved by scanning electron microscopy that the phase-separated marisomes in water were generally much smaller and softer than the original marisome which was phaseseparated in hypertonic solution. Marisome was also solubilized with boiling water, which was phase-separated again by cooling (Fig. 5). The marisome appears to be much softer than the original marisome because the marisome was considerably deformed during drying on a coverslip. Thus these systems were reversible.

The marisome had the percentage composition of carbon $63.67 \%$, hydrogen $6.71 \%$, nitrogen $10.56 \%$, ash $6.54 \%$, and others e.g., oxygen $12.52 \%$. This composition suggests that marisomes mostly consist of organic substances. Judging from solubility for ethanol, sodium dodecylsulfate, and Triton X-100, marisome seems to be made up of lipid-like substances which may have been synthesized from amino acids in the presence of metal ions. By sodium dodecylsulfate and Triton X-100, marisome was completely solubilized but only surface 


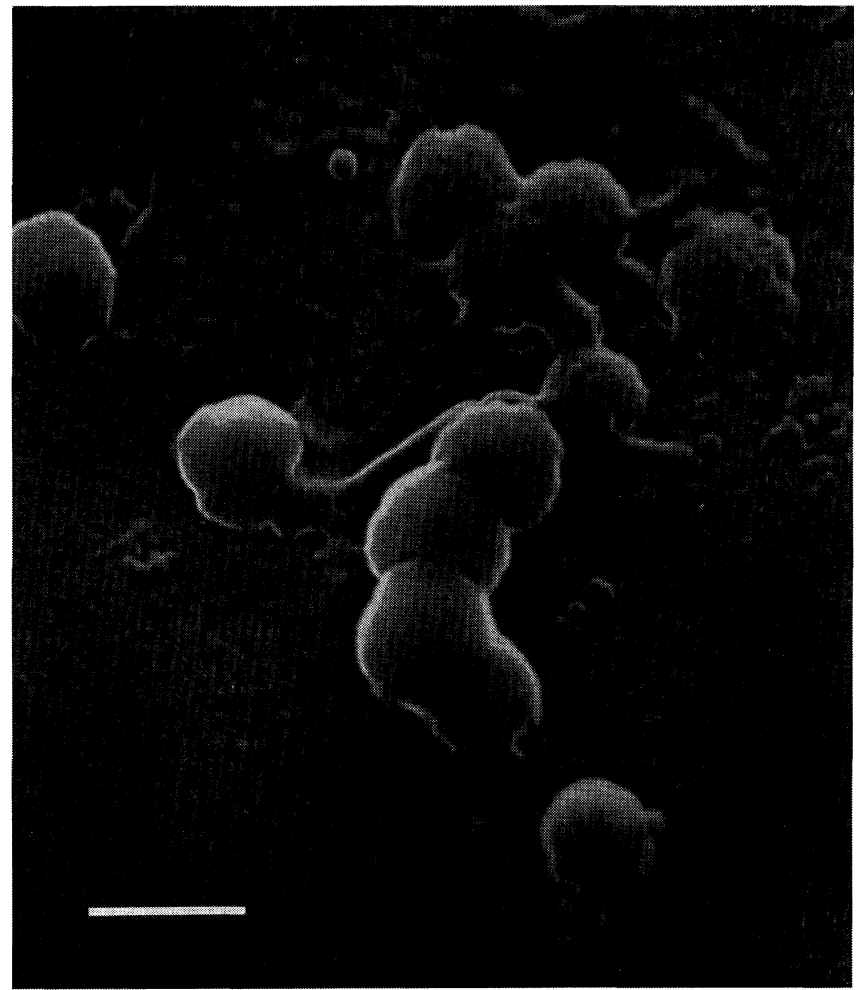

Fig. 5. Scanning electron micrograph of phase-separated marisome by cooling of boiled water solubilized marisome (scale bar, $1 \mu \mathrm{m})$.

structure of marigranules was solubilized. On the other hand, by treatment with alkaline solution, the interior structure of marigranules was completely solubilized but structure of marisome was not appreciably changed. These facts suggest that marisome is a a precursor of marigranule, that is, amino acids, metal ions and condensing agents such as cyanamide and cyanides derived from amino acids may be concentrated in marisome, in which the amino acids may be polymerized to give elastin-like polymers. If marisome is regarded as first phase-separated structure in marine environment and marigranule is regarded as higher-grade structure containing proteins than marisome, the both marisome and marigranules are good models in the course of formation and evolution of protocells. In addition, the modified sea medium was proved to be good environment for a study of chemical evolution by the formation of marisome and marigranule.

Acknowledgements. We thank Mr. S. Kondo in MitsubishiKasei Institute of Life Sciences for scanning electron microscopy and 
for his valuable discussion.

\section{References}

1) Oparin, A. I. (1958): The Origin of Life, Iwanami, Tokyo (in Japanese).

2) Fox, S. W. (1967): Origins of Life, 7, 49.

3) Yanagawa, H., and Egami, F. (1978): Proc. Japan Acad., 54B, 10.

4) - (1978): Origin of Life (Noda, H. ed.), Center for Academic Publication Japan, Japan Scientific Societies Press, Tokyo, pp. 385-390. 